Archives

$26 \mid 2001$

Du cahier de la coutume ... au livre // Études urbaines

\title{
Les interprètes et le droit coutumier
}

Les éditions des coutumes du bailliage d'Amiens

\section{Martine Grinberg}

\section{(2) OpenEdition}

12 Journals

Édition électronique

URL : http://journals.openedition.org/ccrh/1493

DOI : $10.4000 /$ ccrh. 1493

ISSN : $1760-7906$

Éditeur

Centre de recherches historiques - EHESS

Édition imprimée

Date de publication : 20 avril 2001

ISSN : 0990-9141

Référence électronique

Martine Grinberg, «Les interprètes et le droit coutumier », Les Cahiers du Centre de Recherches

Historiques [En ligne], 26 | 2001, mis en ligne le 30 novembre 2008, consulté le 30 avril 2019. URL

http://journals.openedition.org/ccrh/1493; DOI : 10.4000/ccrh.1493

Ce document a été généré automatiquement le 30 avril 2019.

Article L.111-1 du Code de la propriété intellectuelle. 


\section{Les interprètes et le droit coutumier}

Les éditions des coutumes du bailliage d'Amiens

\section{Martine Grinberg}

1 L'étude des éditions des coutumes du bailliage d'Amiens s'inscrit dans une interrogation sur l'histoire du droit et sur les effets de la rédaction des coutumes dans la sphère du droit, plus que sur l'histoire du livre. Cette dernière a donné lieu à de nombreux et d'excellents travaux, bien que le cas spécifique du livre de droit n'ait pas suscité de recherche globale, que le droit coutumier ait été laissé de côté, bien que les rapports qu'entretiennent le texte et son support puissent fournir des indications intéressantes. Les statistiques, que l'on a pu construire, même si elles demeurent approximatives, évaluent la part du livre de droit dans la production imprimée au XvI ${ }^{\mathrm{e}}$ siècle à $4,5 \%-5 \%$. Quelques périodes semblent plus actives pour la production de livres, comme les années 1510-1520 et la première moitié du XVII ${ }^{e}$ siècle, en particulier pour le droit français ${ }^{1}$, mais aussi pour la théologie et l'histoire. Le XvIII ${ }^{e}$ siècle est, lui, plus propice à de grandes entreprises éditoriales de type encyclopédique dont le grand œuvre est, pour les coutumes, constitué par le Nouveau coutumier général de Charles Bourdot de Richebourg ${ }^{2}$ en 1724. La présence des livres de droit dans les bibliothèques ${ }^{3}$ des grands personnages, parlementaires, magistrats, avocats est évidemment plus forte: la bibliothèque de Jacques Auguste de Thou (1553-1617) contient $8 \%$ de livres de droit (canon et civil), celle du chancelier Séguier $8 \%$, de Richelieu $4,1 \%$. Celle du président du parlement de Rouen (1691-1703), Charles-François de Montholon, évaluée à 1500 titres, est une des plus importantes de la Normandie : $43,7 \%$ des titres qu'il possède sont édités au milieu du XVII e siècle, et $40,9 \%$ des ouvrages sont des livres de droit, dont $22,6 \%$ concernent le droit coutumier ${ }^{4}$.

2 Il faut entendre dans la sphère du droit, le droit canon, majoritaire dans la production du livre à la fin du Moyen Âge, le droit romain, le droit coutumier, les livres de pratiques judiciaires. Globalement, la production du livre juridique connait une forte hausse dans les dernières années $d u \mathrm{Xv}^{\mathrm{e}}$ siècle ${ }^{5}$, en Italie, à Venise surtout et à Lyon, qui touche principalement dans un premier temps le droit romain.

3 Le mouvement de rédaction des coutumes, lancé par l'ordonnance de Montils-les-Tours en avril 1454 et précisé par des ordonnances ultérieures ${ }^{6}$, aboutit à l'édition des coutumes 
rédigées et homologuées, sans que l'édition ait été programmée. D'ailleurs, certaines coutumes comme celle de Normandie qui ne fut rédigée et réformée qu'à la fin du $\mathrm{XVI}^{\mathrm{e}}$ siècle (en 1583) fut éditée plusieurs fois auparavant et même avec des commentaires. Ce qui compte avant tout, au plan juridique, c'est le cahier de la coutume déposé au greffe du bailliage et au Parlement, et dont on peut obtenir des copies partielles ou totales. L'édition, qui est indépendante de l'authenticité de la coutume, vient en plus: elle la lance dans le champ public, assure sa diffusion. Certaines éditions ont même été interdites pour cause d'erreurs, comme celles du Berry, de Touraine, celle du Poitou fut contestée par les nobles?

$4 \quad \mathrm{Au} \mathrm{XVI}{ }^{\mathrm{e}}$ siècle, l'édition des coutumes constitue une production spécifique de l'édition provinciale ${ }^{8}$. Pierre Aquilon comptabilise 250 éditions provinciales de coutumiers pour une soixantaine de ressorts. On peut y adjoindre les styles et pratiques destinés aux magistrats, aux notaires, les publications de textes législatifs, lettres patentes, édits royaux et ordonnances. Certains libraires et imprimeurs se spécialisent peu à peu dans ce type de production croissante.

\section{Les éditions des coutumes du bailliage d'Amiens}

Le bailliage d'Amiens est situé dans le ressort du parlement de Paris, et à cette période dans une zone frontière entre le nord du royaume et les Pays-Bas bourguignons et intégré dans le gouvernement de Picardie'. Il est composé de huit prévôtés à la fin du Moyen Âge ${ }^{10}$ : Amiens, Beauquesne (prévôté foraine du costé d'Artois et de la rivière d'Authie), Beauvaisis, Doullens, Foulloy, Montreuil-sur-Mer, Saint-Riquier, Vimeu. Au moment de la rédaction effectuée en 1507, sept prévôtés voient leurs coutumes locales accordées (Beauvaisis, Beauquesne, Doullens, Foulloy, Montreuil-sur-Mer, Saint-Riquier, Vimeu). La réformation de 1567 abroge les coutumes du Beauvaisis et décrète, en plus, les coutumes locales de la ville et prévôté d'Amiens qui n'avaient pu être publiées en 1507 et n'avaient pas été imprimées par faute et omission, mais qui sont néanmoins toujours observées; elle abroge celles du Beauvaisis. À la fin de la période, plusieurs prévôtés sont supprimées par l'édit de septembre 1748 ; le ressort du bailliage présidial d'Amiens se compose dès lors comme suit : la prévôté d'Amiens est supprimée et réunie au bailliage, siège présidial et justice civile d'Amiens, la prévôté de Beauquesne est supprimée. Celle de Beauvaisis avait deux sièges, il ne subsiste que celui de Bourg de Grandvillers. Les prévôtés de Doullens, Foulloy, Saint Riquier et de Vimeu demeurent.

6 Il existe vingt-deux éditions ${ }^{11}$ des coutumes du bailliage d'Amiens entre la première édition, sans date, qui serait de 1508 , donc très rapidement après l'homologation et 1783 , date à laquelle est édité un Traité des donations avec la coutume d'Amiens commentée; ce dernier est édité six fois, entre 1713 et 1783. Sur l'ensemble, on compte une moyenne de six éditions par siècle. Il y aurait en plus, selon le Répertoire bibliographique des livres imprimés en France au seizième siècle ${ }^{12}$, une seconde réédition de celle de 1508, en 1510, par le même imprimeur libraire et un Agrégatoire des coutumes contenant les coutumes générales de la prévôté de Montreuil avec les usages et stile du siège royal (Guillaume Eustace), sans date, mais qui d'après l'adresse serait de 1506, enfin une réédition de la coutume réformée de 1613 en 1623 (à Amiens, par l'imprimerie Jacques Hubault).

7 Sur ces vingt-deux éditions, sept sont sans commentaires ; sur les sept, quatre sont des éditions de la coutume rédigée : 1508, 1516, 1535, 1547. La première et la dernière sont 
éditées à Amiens, les deux autres à Paris. Trois concernent la coutume réformée en 1567. Puis, à partir de 1653, viennent uniquement les éditions de la coutume avec commentaires et ceci jusqu'à la veille de la Révolution. Il faut ajouter à ces éditions le Coutumier de Picardie édité en 1726 qui contient, entre autres, les coutumes d'Amiens, et qui se veut être le premier volume d'un Recueil de commentateurs, entreprise éditoriale contemporaine du Nouveau coutumier général, publié par Charles Bourdot de Richebourg.

Le nombre d'éditions des coutumes d'Amiens n'est pas très important comparé à d'autres coutumes (Normandie ou Bourgogne par exemple), mais il est clair que l'intérêt pour le droit coutumier ne faiblit pas, que ce dernier suscite tout au long de la période de nouveaux commentaires qui prennent en compte l'évolution du droit et de la jurisprudence. Il faut, en outre, adjoindre à ces éditions spécifiques de la coutume du bailliage d'Amiens, celles des coutumiers généraux qui débutent en 1517 et se terminent en 1724-1729 avec le livre de Charles Bourdot de Richebourg : au total trente éditions ${ }^{13}$.

9 Sur ces vingt-deux éditions, certaines semblent être des rééditions, ainsi celle (coutume rédigée) de 1535 est identique à celle de 1516, imprimée pour le même libraire de Paris (Guillaume Eustace), celle de 1571 (coutume réformée) à Paris pour Jean Dallier serait rééditée à Paris pour un autre libraire en 1575 dans le même format et dans un autre format en 1613 à Amiens. Celle du commentaire de Du Fresne de 1662 est édité pour deux libraires et rééditée en 1671 par l'un des deux et réintégrée dans le Coutumier de Picardie; les coutumes commentées par Adrien de Heu sont éditées une fois en 1653 et reéditées dans le même coutumier. Les œuvres de Jean-Marie Ricard ont plus de succès encore : quatre éditions de la coutume commentée, avec ajouts successifs, plus sa reproduction dans le Coutumier de Picardie, et six éditions de son Traité des donations avec la coutume d'Amiens, qui fut donc un vrai succès de librairie au cours du XviII ${ }^{\mathrm{e}}$ siècle.

10 Enfin, derniers prolongements de l'édition au XIX siècle, des érudits puisent dans les archives des coutumes qui n'ont jamais été éditées auparavant: les Coutumes locales du bailliage d'Amiens rédigées en 1507, celles qui n'ont pas été reçues en raison de leur trop grand nombre lors de l'assemblée de rédaction, mais qui sont conservées dans les archives du bailliage, et l'ancien coutumier inédit de Picardie du début du XIV siècle $^{14}$.

11 En ce qui concerne le format, les quatre premières éditions des coutumes rédigées sont in $-8^{\circ}$ et en caractères gothiques (de 1508 à 1547). Dans l'ensemble de la production imprimée, le gothique disparaît à partir des années 1530 pour laisser place aux caractères romains et italiques. Un seul commentaire est in $-8^{\circ}$, celui de 1734 . L'édition de la coutume réformée en 1567 abandonne l'octavo pour le quarto et une in-16. Quant aux commentaires, sur quinze éditions, dix sont des folios, trois in- 12 , une in $-8^{\circ}$ et une in $-4^{\circ}$. Le public auquel s'adresse ces livres et la valeur du livre changent. Les folios, particulièrement appréciés des magistrats et des juristes comme on peut le constater dans leurs bibliothèques, se multiplient à partir $\mathrm{du} \mathrm{XVII}^{\mathrm{e}}$ siècle, surtout pour les livres liturgiques et les livres de droit.

Les lieux d'éditions confirment la prédominance parisienne. 


\begin{tabular}{|l|c|c|c|}
\hline & $\begin{array}{c}\text { Sans } \\
\text { commentaire }\end{array}$ & $\begin{array}{c}\text { Avec } \\
\text { commentaire }\end{array}$ & $\begin{array}{c}\text { Coutunnier } \\
\text { Picardie }\end{array}$ \\
\hline Amiens & 3 & & \\
\hline Paris & 4 & 13 & 1 \\
\hline $\begin{array}{l}\text { Clermont- } \\
\text { Ferrand }\end{array}$ & & 1 & \\
\hline Abbeville & & 1 & \\
\hline
\end{tabular}

$12 \mathrm{Au}$ total, deux éditions de la coutume rédigée sont éditées à Amiens, une seule de la coutume réformée, ce qui est peu sur l'ensemble des éditions et pose la question de la présence et de l'activité des libraires imprimeurs amiénois.

Amiens est une grosse cité drapière de 15000 à 20000 habitants environ à la fin du Xvi siècle. Elle est réunie à la Couronne de France en 1471, reprise pour une brève période par les Espagnols en 1597. Elle n'a pas de cour souveraine, ni d'université, ce qui explique en partie peut-être le nombre relativement faible de libraires et d'imprimeurs. Ce constat s'applique aussi à l'ensemble de la Picardie qui souffre sans doute de la proximité avec Paris $^{15}$. La première imprimerie dans la région date de 1487 , mais elle est située à Abbeville ; elle imprime quatre ou cinq ouvrages (dont la Cité de Dieu et la Somme rural de Jehan Boutillier) et disparaît. En ce qui concerne Amiens, on signale des loges près de la cathédrale dès 1437 , une douzaine fin $\mathrm{XVI}^{\mathrm{e}}$ siècle dont huit libraires. Ce sont d'abord les écrivains et les copistes qui occupent les lieux. Les premiers libraires apparaissent dans les premières années $\mathrm{du} \mathrm{XVI}^{\mathrm{e}}$ siècle. Leur production est faible, et beaucoup de libraires font imprimer à Paris.

14 Nicolas le Caron, imprimeur libraire, édite, d'après Albert Labarre, deux livres, en 1508 et en 1510 : les coutumes du bailliage. L'édition supposée de 1508, car le procès-verbal de la rédaction est daté d'octobre 1507, est imprimée sur l'ordonnance des commissaires, ce qui la classe si l'on suit John Gilissen ${ }^{16}$ dans la catégorie des éditions officielles. Le Caron y adjoint l'ordonnance de Louis XII de 1507 pour les universités, les monnaies, les orfèvres. Ce Nicolas Le Caron, cité comme sergent au bailliage en 1510, puis procureur du roi dans un document de 1515, devient greffier au bailliage en 1520 et meurt vers 1524 . Une carrière brève comme imprimeur libraire. Trois autres libraires sont connus jusqu'à Jean Le Caron, qui édite à nouveau ces mêmes coutumes en 1547, cette édition servira (et non le cahier) à l'assemblée de réformation de la coutume en 1567; le procès-verbal la mentionne précisément.

C'est avec Jacques Hubault que l'imprimerie reprend définitivement à Amiens, en 1609. Dès le début il se heurte à Adrien Delaunay venu de Rouen pour la fourniture du collège des jésuites, grand consommateur de livres. L'échevinage décide que Delaunay vendra ce que Hubault imprimera. Trente-neuf impressions sont connues. L'inventaire après-décès signale 11931 volumes, dont beaucoup sont des livrets de faible valeur, des ouvrages imprimés par lui dont 250 volumes de la coutume du bailliage prisée 3 sous pièce, 4 sous pour un exemplaire relié. C'est lui qui imprime la coutume réformée en 1613, quarante ans après l'édition officielle de 1571 (Paris, pour Jean Dallier). Sur les 253 titres édités à Amiens jusqu'en 1788 , on recense trois éditions des coutumes du bailliage d'Amiens, deux 
de celles du Ponthieu et une de la coutume locale d'Abbeville. À la suite de Jacques Hubault, vient Robert Hubault (1634-1657), libraire et imprimeur du roi, qui imprime dixhuit publications entre 1639 et 1656. Puis la veuve Robert Hubault (1657-1697) est signalée à Paris lorsqu'elle publie en 1683 les coutumes du bailliage d'Amiens commentées par Jean-Marie Ricard, déjà publiées en 1661 par Louys Billaine à Paris.

Le premier libraire parisien de la coutume du bailliage d'Amiens (2 éditions) est Guillaume Eustace, libraire du roi et relieur juré, nommé libraire juré en 1509. Jean Dallier fait imprimer en 1571 la coutume réformée; il est imprimeur et libraire et obtient le privilège royal pour les publications des ordonnances sur les monnaies. C'est lui également qui publiera la coutume de Touraine réformée. Certaines éditions de la coutume commentée, plus onéreuses que la coutume seule, mentionnent deux ou plusieurs libraires associés dans l'opération, celle de 1662 du commentaire de Jean Du Fresne porte deux noms (Michel Bobin et Nicolas le Gras), les mêmes pour la réédition de 1671. Celle qui contient le commentaire de Jean-Marie Ricard, de 1712, cite quatre noms : Guillaume Cavelier, Michel Guignard et Charles Robustel, Nicolas Gosselin. Les lettres de privilège expliquent la procédure; que ce soit l'auteur ou le libraire qui sollicite les lettres de privilège, il peut céder tout ou partie de ses droits à un ou plusieurs libraires. Ainsi, pour l'édition des œuvres de Jean-Marie Ricard, demandée par son fils, celui-ci cède les droits à Seneuze qui, lui-même, en cède la moitié à un autre libraire Jean Guignard (1682). Pour la réédition du Traité des donations avec la coutume d'Amiens en 1713, le libraire cède la moitié de ses droits à deux autres. Le Coutumier de Picardie s'inscrit dans un type de projet plus ambitieux, comme d'autres au cours du XVIII siècle; l'avocat anonyme qui souhaite faire imprimer l'ouvrage obtient les droits en 1722 et les cède à huit libraires parisiens. Ce coutumier présente sur la page de titre la formule Paris, aux dépens de la société. L'avocat au parlement de Paris, dont on tait le nom, a souhaité faire imprimer ce recueil de commentateurs, après un long travail de recherche. En raison des frais prévisibles, et de peur que d'autres s'avisent de contrefaire cet ouvrage, il demande des lettres de privilège qu'il obtient : pour 20 ans, droit de vendre et faire vendre, à condition qu'il ait fini d'ici 12 ans, et qu'il en publie un volume chaque année.

17 La première édition des coutumes d'Amiens avec privilège du roi date de 1571. Apparu à la fin du Xv $v^{e}$ siècle à Lyon, le privilège prend des formes variées ${ }^{17}$, la durée peut aller de 6 mois à 10 ans; il peut être cédé à un confrère. La législation sur le livre se met en place tout au long de la période, le pouvoir royal contrôle la production et définit les conditions d'exercice des métiers associés au livre. À partir de 1566 (ordonnance de Moulins), aucun livre nouveau ne peut être édité sans autorisation du roi accordée sous forme de lettres de privilège sous notre grand scel, donc sous contrôle du chancelier; d'abord soumis à des experts, puis à la chancellerie pour approbation, le livre peut enfin obtenir des lettres de privilège. Pour certains ouvrages, à partir du début du XVII siècle, le régime du privilège ne s'applique pas et seule la permission d'imprimer est nécessaire ${ }^{18}$. Toutes les éditions de la coutume réformée du bailliage d'Amiens portent mention du privilège sauf celle de la coutume de 1613 et celle de l'édition commentée par Jean-Marie Ricard publiée à Abbeville en 1781 qui mentionne seulement avec permission.

18 Ces lettres de privilège fournissent un certain nombre d'indications, à partir de l'édition de 1653 (édition commentée par Adrien de $\mathrm{Heu}$ ). D'abord elles indiquent la durée du privilège, neuf ans pour le commentaire d'Adrien de Heu, sept ans pour celle de JeanMarie Ricard, mais 20 ans pour le Traité des donations avec la coutume d'Amiens, 20 ans aussi pour le Coutumier de Picardie. Elles informent aussi sur l'initiateur du projet d'édition : le 
marchand libraire Gervais Alliot souhaite faire imprimer le commentaire d'Adrien de Heu, le libraire Jean Guignard fait la demande pour le Traité des donations de Jean-Marie Ricard, en 1713, en revanche c'est Jean Du Fresne, l'auteur du commentaire qui fait la demande, ou le fils de l'auteur décédé (Ricard) qui souhaite faire rééditer sous son contrôle tous les ouvrages de son père en y intégrant des nouveaux commentaires tirés de ses manuscrits (accordé en 1682). L'édit d'août 1617 décide d'accorder le privilège à condition de déposer deux exemplaires à la Bibliothèque publique. Cet élément est effectivement mentionné dans les éditions de coutumes: don obligatoire à notre bibliothèque publique, un exemplaire à celle du Louvre, un autre à celle du garde des sceaux. Au XVII ${ }^{\mathrm{e}}$ siècle, le privilège est de plus en plus souvent reconduit, ce qui favorise certains libraires, à partir des années 1650, et entraîne la limitation voire la disparition du domaine public. La suppression des privilèges provinciaux est décidée en 1678 . Tout cela pénalise la production du livre et particulièrement provinciale, alors que la demande croît $^{19}$, et favorise l'édition étrangère et l'édition frauduleuse. Les imprimeurs du roi sont favorisés car ils sont assurés de garder une part du marché, l'édition de plus en plus massive des actes officiels. Par ailleurs, les grandes institutions grandes productrices de livres ont leurs libraires et imprimeurs spécifiques, qu'elles soient religieuses ou étatiques, universités, parlements, collèges de jésuites, évêchés, etc.

Une des éditions commentées de la coutume d'Amiens évoque un autre aspect du problème : les rapports entre libraires et auteurs. Des éditions qui semblent être le fruit d'initiatives privées des libraires montrent peu de respect pour le texte de l'auteur. Ainsi dans sa préface à l'édition de 1661 des Coutumes du bailliage d'Amiens, avec les notes de Charles du Moulin et autres remarques de Jean-Marie Ricard, ce dernier, ayant beaucoup travaillé sur la coutume de Paris, déclare que ses observations ont été imprimées contre sa volonté et sur un exemplaire qu'il n'avait point revu, puis qu'il a travaillé sur les coutumes de Senlis, d'Amiens à la suite de du Moulin, et qu'on a omis dans toutes les éditions parues jusque-là plus de la moitié des notes de ce dernier. La crainte des auteurs de voir paraître des éditions de leurs travaux de mauvaise qualité et des contrefaçons est une des raisons qui les pousse à solliciter des lettres de privilège ${ }^{20}$.

\section{Le livre en mouvement : l'accès au texte}

L'imprimerie ne modifie pas radicalement la morphologie du livre; l'héritage médiéval persiste quelque temps, puis de nouveaux outils, une nouvelle lisibilité s'élaborent. La scolastique médiévale a déjà constitué l'essentiel de l'appareillage textuel, tables, divisions, sommaire, index, qui sera réinterprété et amplifié dans le nouveau support imprimé et tend vers une certaine uniformisation dans le cadre d'une production en série et d'une plus grande diffusion. L'évolution est progressive qui différencie peu à peu le livre imprimé du manuscrit médiéval.

\section{La page de titre}

21 Un des premiers éléments de cette différenciation est constitué par l'apparition et l'élaboration de plus en plus soignée de la page de titre, très rarement présente dans le livre médiéval. La page est mise en espace, elle se couvre d'éléments divers, l'ornementation, la marque du libraire, les gravures, le nom et l'adresse du libraire et/ou de l'imprimeur. 
22 Les pages de titre des éditions de coutumes suivent bien sûr l'évolution générale. La première édition de 1508 reste très proche du livre médiéval : le titre est construit comme un pavé de onze lignes, mais de même typographie que le reste du texte. Dès la seconde édition de 1516, la page est ornée d'une marque, identique en 1535. En 1547, l'apparition $\mathrm{du}$ titre en triangle isocèle, pointe en bas, avec caractères décroissants et coupures de mots curieuses met en valeur la géométrie du texte plus que le sens : Coustumes ge/neralles du bailliage d'Amiens avec celles des/prévostés... En 1571, le titre est un pavé rectangulaire : Coustumes/tant gene/rales que loca/les et particulières du/bailliage d'Amiens, mises et re/digées par escript de l'advis des trois états dudict bail/liage, par nous... Typographie variée, mélange des corps et des styles, couleur - rouge et noir (à partir de l'édition de 1653) -, tous ces éléments se perfectionnent pour faire de cette page une vitrine séduisante du livre.

Les premières éditions donnent l'intitulé exact des coutumes générales avec celles des prévôtés dont la liste suit, celles de la coutume réformée reprennent évidemment le changement de l'intitulé décidé à l'assemblée de réformation (Coutumes tant générales que locales et particulières du bailliage d'Amiens...) et portent les noms des commissaires chargés de la réformation avec leurs titres respectifs. Les commissaires sont mentionnés, mais pas nommés dans la page de titre dans les quatre premières éditions $(1508,1516,1535,1547)$. En revanche dans l'édition officielle de la coutume réformée de 1571 et dans celle de 1613, les noms sont accolés au titre, ils en font partie. Puis ils disparaissent des titres des éditions avec commentaires.

Le livre imprimé est encore très proche du cahier de la coutume au XVI siècle. La référence au bailliage est plus forte dans le nouvel intitulé décidé à la réformation qui fait disparaître le nom des prévôtés; alors que les coutumes locales et particulières n'apparaissaient pas dans le titre en 1507, elles se trouvent désormais incluses dans le cadre englobant du bailliage et perçues plus nettement comme dérogeantes à la coutume générale, lors de la réformation. Les éditions de commentaires prennent de la distance avec le texte de droit: le titre stipule seulement la coutume générale du bailliage d'Amiens sans citer les coutumes locales ou particulières (Adrien de Heu), voire même Coutume d'Amiens commentée sans mention du bailliage (pour celle qui suit le Traité des donations). Elles insistent sur d'autres points: la page de titre indique les noms des commentateurs, leur fonction, leurs titres, la présence d'une table des matières, le contenu des textes associés à celui de la coutume, les questions de droit, les arrêts, la conférence avec d'autres coutumes, etc. Bref, elles enserrent le texte de la coutume dans un réseau beaucoup plus vaste de relations. Le titre est l'équivalent d'un sommaire. Le Coutumier de Picardie présente deux pages de titre, 1) Recueil des commentateurs anciens et modernes sur les coutumes générales et particulières de France avec leurs textes corrigés; 2) Coutumier de Picardie contenant les commentaires de... Suivent les noms des commentateurs successifs de la coutume du bailliage d'Amiens, ceux des autres coutumes de Picardie, et la mention, détachée de ce qui précède, avec des questions importantes sur plusieurs articles des mêmes coutumes, traitées par les plus célèbres avocats au Parlement.

Ces variantes du titre, par rapport au cahier de la coutume réformée, s'accompagnent aussi d'un tri dans le texte global de la coutume. Ainsi Adrien de Heu choisit de ne commenter que la coutume générale du bailliage, et pas les coutumes locales pourtant initialement accolées à la coutume générale. Jean Du Fresne commente la coutume générale du bailliage et une seule coutume locale, celle de la ville d'Amiens. Ces deux auteurs présentent leur livre d'abord comme un commentaire. En revanche Jean-Marie 
Ricard formule l'intitulé exact de la coutume suivi des noms des commentateurs : notes de du Moulin, remarques de Jean-Marie Ricard. Les interprètes, dans ce cas, sont en position de déférence par rapport à l'écrit de droit, ils sont au service du texte.

Cette présentation inscrit les juristes comme metteurs en lumière du texte, et souligne leur effacement relatif en tant qu'auteurs. Ils sont avant tout débiteurs, ils ont à rendre compte de l'esprit des lois (mens juris, selon l'expression de Bartole), à traduire, à transmettre le sens, pour atteindre la vérité du texte jamais complètement dévoilée. Cette dette sans fin justifie l'accumulation de commentaires, de références aux autorités, à la jurisprudence ; le titre du Nouveau coutumier général de Charles Bourdot de Richebourg en est un exemple parfait ${ }^{21}$.

Le Traité des donations associe une des questions traitées par le droit coutumier, en intégrant l'apport de plusieurs coutumes dans une réflexion théorique, avec la coutume d'Amiens placée presque en annexe du texte. Le titre de l'édition d'Abbeville de 1781 a, elle, supprimé les termes générales et locales: on insiste moins sur la diversité des coutumes à l'intérieur du ressort comme on le faisait au temps des rédactions, et plus sur l'unité. Enfin, le Coutumier de Picardie est le premier volume d'un Recueil des commentateurs anciens et modernes sur les coutumes, et non un recueil des coutumes (contrairement à l'entreprise de Bourdot de Richebourg). Ces deux entreprises rassemblent les divers commentaires déjà édités auparavant en les augmentant de nouvelles remarques et d'éléments de jurisprudence qui servent à l'interprétation des coutumes. Si les interprètes de la coutume hésitent à se considérer comme des auteurs, les coutumes, elles, sont bien entrées dans la sphère de l'interprétation au sens juridique du terme. Elles font désormais partie de la tradition, et l'interprétation se doit de l'enrichir.

\section{Sommaires et tables des matières}

29 Ces éléments, mis en place aux XII ${ }^{\mathrm{e}}$-XIII ${ }^{\mathrm{e}}$ siècles, ne sont donc pas nouveaux, mais ils vont se développer pour faciliter la lecture et l'accès au texte, induisant un type d'approche et d'usage du livre particulier, comme un outil de travail.

Seule la première édition n'a pas de sommaire ; elle commence par le procès-verbal suivi de la coutume rédigée, suivie du décret des coutumes, puis les coutumes générales gardées au lieu du siège royal du bailliage d'Amiens à Montreuil et des autres - celles qui seront appelées locales à la réformation. Le livre se termine par l'ordonnance de Louis XII et le répertoire pour trouver facilement les matières avec renvois aux pages. En 1516 et en 1535, le sommaire est présent, mais sans renvoi aux pages, il cite les lieux et la coutume du lieu. Il faut attendre 1547 pour que le procès-verbal apparaisse en tant que tel dans le sommaire, distingué du texte de la coutume, et pour que la pagination soit indiquée dans le sommaire. Ce dernier s'affine en 1571 (table des rubrices, placée après l'épître en latin) : les titres de la coutume générale et des coutumes locales sont indiqués par ordre alphabétique, comme l'enseignait la scolastique, avec pagination. C'est un outil de travail, où la rationalité fait violence à l'ordre du texte. Les coutumes locales sont citées, sous un titre général qui n'est pas exact coutumes locales de la ville, loy, mairie, prévôté, échevinage et banlieue, d'Amiens/de Beauquesne/etc., par ordre alphabétique sans autre mention du fait qu'elles soient locales ou particulières (alors que le texte de la coutume le précise). Puis vient le sommaire du procès-verbal qui reprend les titres de la coutume toujours dans l'ordre alphabétique, puis celui des coutumes locales. Le livre se clôt par l'extrait des registres du Parlement. La présentation est identique dans l'édition amiénoise de 1613. 
31 À partir de 1653, date du premier commentaire, plusieurs éléments viennent s'interposer entre la page de titre et le sommaire. Celui-ci suit les titres de la coutume avec renvoi aux pages. Ce commentaire d'Adrien de Heu ne contient pas de procès-verbal, à croire qu'il n'est plus nécessaire: la preuve de la coutume est faite depuis longtemps; le commentateur se livre à l'interprétation. En revanche, la table des matières revient, très détaillée qui renvoie aux pages, aux notes, aux articles de la coutume ou au commentaire, signalant par un pied-de-mouche, une main ou une étoile, les divers commentaires qui se sont succédé. Les matières sont formulées sous forme de questions, souvent associées à un terme: par exemple Testament suivi de tout ce qui a trait au testament. On peut y repérer le travail qui s'effectue dans l'organisation du savoir juridique; soit sur la langue, modernisée, par rapport à celle des coutumes qui semble de plus en plus archaïque aux juristes des $\mathrm{XVII}^{\mathrm{e}}$ et XVIII ${ }^{\mathrm{e}}$ siècles ; soit par un travail sur les catégories, en faisant émerger peu à peu de la masse des matières citées des mots clés, qui rassemblent des sousensembles établissant ainsi une hiérarchie dans les matières du droit coutumier. Des renvois sont faits à d'autres termes, ainsi pour Fiefs, il faut aller chercher aussi à Succession, Retrait, Droits seigneuriaux, ou pour savoir ce Que signifient ces mots de foy et hommage, on cherchera à Fiefs,. La construction du champ sémantique et des collocations des notions juridiques, la mise en place progressive de ce qui ressemble à un thésaurus sont repérables dans l'analyse de ces tables ${ }^{22}$. La question citée quel âge il faut avoir pour pouvoir tester (de Heu), devient age pour pouvoir faire testament, voyez Testament, dans la table de Du Fresne (neuf ans plus tard). Le procédé de renvoi est un peu plus développé dans le commentaire de Du Fresne. Au terme vassaux, il est écrit de se rendre à seigneurs de fiefs, lettre $S$ et à saisie féodale, lettre $S$. Rien n'est cité à la lettre $G$ dans cette table, alors que celle d'Adrien de Heu mentionne garde noble, avec renvoi à Fiefs, et Gage, avec renvoi à Hypothèques. La typographie différenciée et la mise en page rendent cette évolution plus lisible encore (dès l'édition de 1661 des coutumes commentées par Jean-Marie Ricard) : les mots clés sont en italiques, tout ce qui en dépend est en retrait et en caractères romains.

Au terme Fiefs (édition de 1712, du commentaire de Ricard), la question comment se partagent entre cohéritiers est renvoyée à Succession. Faut-il y voir le signe d'une tendance à renforcer certaines catégories du droit civil au détriment du droit féodal en tant que tel ? $C^{\prime}$ est ce que Jean-Baptiste Domat ${ }^{23}$ dans son grand livre théorique Les loix civiles dans leur ordre naturel a formulé explicitement et d'une manière radicale. Toutes les matières du droit ont un ordre naturel. Les matières se divisent en deux parties, les engagements et les successions. En conséquence, pour ce qui est des matières qui sont propres à nos coutumes comme sont les fiefs, le retrait lignager, la communauté de biens entre le mary et la femme... il est facile de juger que ces matières se rapportent ou aux engagements ou aux successions ${ }^{24}$. À suivre Domat, la table des matières des coutumes serait considérablement simplifiée. Un certain nombre de commentateurs, sans aller aussi loin, ont fait émerger, en mettant de l'ordre dans la masse des articles de coutumes, dans leur diversité, quelques catégories clairement identifiables du droit civil commun, et ont tenté d'inscrire le droit coutumier dans une nouvelle architecture du droit.

\section{Les à-côtés du texte de la coutume}

À part l'ordonnance de Louis XII intégrée dans l'édition de 1508, il faut attendre 1571 pour voir apparaître un autre texte que celui de la coutume dans le livre : l'Épitre à Christofle de Thou en latin, langue des juristes encore et qui donne de l'autorité au texte, 
parle de l'antiquité des lois, de Lycurgue et Solon, de la loi salique, de Rome...; elle est reprise dans l'édition de 1613 d'Amiens. Les éditions suivantes, en mettant à part les commentaires, adjoignent au texte de la coutume et à celui des interprètes d'autres éléments, qui font du livre un objet précieux et qui l'inscrivent dans un réseau de relations et de protection. Le livre trouve enfin sa justification dans l'attente supposée du public.

\section{La dédicace}

La dédicace du livre posthume d'Adrien de Heu (1653) est au verso de la page de titre et commence par le portrait gravé de Louis de Valois à qui est dédié l'ouvrage, puis page suivante vient le texte : A très haut et puissant prince, Louis de Valois, duc d'Angoulême, pair de France, comte de Ponthieu; la dédicace est signée Nicolas de Boulongne, conseiller et procureur du roi au présidial d'Abbeville.

Elle est suivie d'un texte en latin : in commentaria ad consuetudines ambianenses, où il est expliqué qu'il s'agit d'un travail posthume d'Adrien de Heu. Suivent la table des titres contenus dans le livre, le privilège du roi et enfin la coutume commentée.

La dédicace du commentaire de Jean Du Fresne $(1662,1671)$ est adressée à Monseigneur messire Guillaume Lamoignon, chevalier seigneur de Bâville, baron de S. Lyon et de Poissy, conseiller du roi en tous ses conseils et premier président en sa cour de Parlement, et signée de Du Fresne. Les termes en sont d'un lyrisme et d'une déférence tels, vis-à-vis de la figure de l'autorité et de la souveraineté, qu'ils méritent d'être rapportés :

Puis que c'est un ordre inviolable en la Nature, que toutes les choses retournent facilement à leur principe, et que par iceluy les Astres s'estans levez au point du jour retournent par l'Occident à l'Orient, et que les fleuves prenans leur cours de la mer y refluent avec pareille vitesse qu'ils en sont partis, l'on ne jugera pas que c'est par vanité que je vous dédie cet ouvrage, pour paraître à l'éclat de votre Pourpre et de l'Autorité qui vous environne...

La dédicace existe dans d'autres types d'ouvrages, elle est le fait du libraire ou de l'auteur, elle sollicite la protection de celui à qui elle s'adresse, et place donc l'auteur en situation de soumission. Les juristes sont d'une certaine manière encore plus redevables, ou du moins autrement, car ils œuvrent dans le champ du pouvoir, tirent leur propre justification et reçoivent leurs lumières de l'Autorité suprême ${ }^{25}$.

Dans cette édition de Du Fresne cinq épigrammes suivent la dédicace, trois en latin, deux en français : de Du Fresne, de Cousin, de du Quesnoy (avocat) et deux en français en l'honneur de l'auteur. Celui qui signe L.S.F. confirme ce que la dédicace laissait supposer du rôle social et de la place de l'interprète :

Du Fresne en composant ce docte commentaire

$\mathrm{Tu}$ fis sans doute plus que tu ne voulais faire

Ton autorité (sous celle de ton prince)

Fait passer tes écrits pour loys dans ta province.

En 1685, le Traité des donations de Jean-Marie Ricard est dédié par Seneuze, libraire, à messire Louis-François Lefèbvre de Caumartin, chevalier, conseiller d'État ordinaire, qui fut intendant en Champagne, commissaire du roi en Bretagne et qui lutta contre les usurpations de la noblesse. 


\section{L'adresse au lecteur}

40 lecteur dans quelques éditions, de l'auteur au lecteur dans d'autres, en tout cas la prise en compte du public est évidente : l'auteur ou le libraire vante les mérites de l'ouvrage offert au public. Le commentaire de Jean Du Fresne, qui est le second des coutumes du bailliage d'Amiens ( 9 ans après celui d'Adrien de Heu), présente aux dires de l'imprimeur des avantages par rapport au précédent : 1) l'auteur est avocat et donc très au courant de la jurisprudence ; 2) il commente la coutume locale de la ville d'Amiens, ce qui n'a pas encore été fait ; 3) il publie 25 arrêts servant de preuve et éclaircissements aux difficultés qu'il a agitées; 4) il explique de nombreux articles par forme de conférence avec les coutumes voisines (qui sont pays de nantissement et de vest et devest) ; 5) il édite l'édit d'Henri IV sur la police de la ville d'Amiens pour apprendre par forme d'histoire. Un ensemble de raisons à valeur pédagogique qui englobe la jurisprudence, où les arrêts contradictoires, et pas obligatoirement du ressort de la coutume, suscitent de nouveaux commentaires. Le texte de la coutume sert ainsi de support à une réflexion théorique globale.

41

La préface du commentaire de Jean-Marie Ricard de 1661, qu'il a rédigée lui-même, fournit d'autres raisons en des termes que l'on retrouve souvent parmi les juristes : il a beaucoup travaillé sur la coutume car c'est la partie principale de la jurisprudence, il décide de donner au public le résultat de son travail en choisissant de ne s'intéresser qu'aux difficultés. Les rééditions qui accompagnent le Traité des donations contiennent une précision donnée par une tierce personne, juriste, qui explique l'ajout de nouveaux commentaires à ceux de Jean-Marie Ricard :

[...] la proximité de la coutume d'Amiens qui régit une partie du bailliage de Beauvaisis m'a donné lieu à éclaircir plusieurs difficultés, ou que nous avons eu à juger ou qu'on m'a proposées, ou que je me suis fait à moi-même....

Il complète donc les commentaires de Ricard par les siens - après en avoir conféré avec Ricard et Du Fresne - repérables typographiquement dans l'ouvrage par un pied-demouche. On y voit la manière dont le droit se construit par interprétations successives, identifiables, parfois contradictoires. Le souci de rendre compte de toute nouvelle interprétation, qui émergerait de la jurisprudence, fait aussi partie du processus. C'est la raison pour laquelle on (son fils sans doute) a récupéré, après la mort de Ricard, ses réflexions portées dans le journal manuscrit qu'il tenait de ses activités au palais de justice, rassemblé ses fragments de notes; on y a joint les dernières décisions des cours souveraines, qu'elles soient conformes ou non à ses avis. Il est possible aussi d'imaginer le tourment que provoque cette quête sans fin chez certains juristes à lire l'avertissement situé en tête du Traité des donations qui passe pour un chef-d'œeuvre de la profession, les juges s'en servent, et même les Parlements qui suivent pourtant la disposition du droit écrit ont de l'estime pour ce travail. L'auteur fut très apprécié pour ses jugements, mais ses trop grandes lumières l'empêchaient souvent de se déterminer dans ses doutes, au point qu'il plaidait difficilement et changeait d'opinion. N'importe, toutes ses réflexions, y compris les hésitations, seront consignées et données au public.

Une dernière raison peut être invoquée pour l'édition d'un ouvrage : la rareté, au sens où il est difficile de se le procurer, et qu'il est censé manquer au public. Ce qui laisse supposer une demande croissante au XVIII ${ }^{\mathrm{e}}$ siècle, puisque le Coutumier de Picardie de 1726 et le commentaire de Jean-Marie Ricard publié26 en 1781 invoquent cet argument dans leurs préfaces respectives. La rareté des coutumiers est, d'après le Coutumier de Picardie, 
[...] venue à un point qu'à peine la vie d'un homme suffit pour les rassembler dans un même corps de bibliothèque : d'ailleurs elle en a rendu le prix si extraordinaire que tel commentateur qui se donnait, il y a dix ans, pour six livres, se vend aujourd'hui trente, d'autres ne se trouvent que très difficilement, ou après un long espace de temps.

y a donc pénurie, explique l'avertissement du Coutumier de Picardie, et c'est ce qui a engagé une compagnie de libraires de Paris à les faire réimprimer répondant ainsi à la demande de l'avocat qui s'est chargé de la conception de l'ouvrage et qui a obtenu les lettres de privilège. La compagnie de libraires, avec Gosselin en tête, signe la dédicace à Monseigneur d'Armenonville, garde des Sceaux, commandeur des ordres du roi, et rappelle que le recueil des commentateurs de toutes les coutumes du royaume se trouve épars en tant de volumes, de formes si différentes et si difficiles à rassembler qu'à peine se trouvait-il complet dans quelques cabinets de savans, alors qu'il est indispensable à ceux qui se destinent aux fonctions de la magistrature et du barreau. Si cette entreprise peut être utile à la République des Lettres, elle mérite la protection du prince.

Le projet doit aboutir à la publication de vingt-et-un coutumiers de province qui se vendront séparément. Un soin particulier a été apporté à la correction des fautes dans le texte de la coutume et dans les commentaires, au choix du papier, et à la qualité de l'impression. Pour la première fois, dans les éditions de la coutume du bailliage d'Amiens, est donnée une table des lieux régis par la coutume. La recherche de la perfection de l'objet est en parfaite harmonie avec l'idée que les juristes se font du droit: un art. Il convient que le support du texte soit digne de ce dont parlaient certains commentateurs, la beauté du droit - parce que les questions de droit sont belles en elles-mêmes affirme Boullenois, ou comme l'exprime Bouhier parce que si l'on considère la connaissance des loix du côté de la beauté, $j$ 'ose dire qu'en cela elle ne le cède à aucune autre ${ }^{27}$.

À la fin du volume se situe l'Approbation : j'ai lu par ordre du garde des sceaux... Je n'y ai rien trouvé qui puisse en empêcher l'impression, au contraire, cet ouvrage est en mesure de parfaire l'instruction et la connaissance du Droit français du public.

C'est à quoi aboutit le lent et patient travail des interprètes, depuis les rédactions de coutumes, dessiner les contours d'un Corps du droit français, lui donner forme et autorité.

\section{Les prolongements de la glose}

La rédaction de la coutume pose d'une nouvelle manière la question de la hiérarchie des sources du droit. La mise en écrit la destinait à devenir loi perpétuelle. La coutume rédigée n'a plus besoin dès lors d'être prouvée. Mais à lire les commentateurs, il est clair que la coutume n'est pas exactement l'équivalent de la loi. Certains cependant comme Guy Coquille (1523-1603) pensent que les coutumes sont le vrai droit civil et peuvent être appelées Droit écrit ${ }^{28}$, terme réservé pour d'autres au droit romain. Une hiérarchie des sources de droit demeure, même si elle s'atténue au cours de l'époque moderne. Au sommet, voire au-dessus, le droit romain - Raison écrite - est à l'origine des principes du droit, toujours la référence quand la coutume est silencieuse. Puis viennent le droit canon, les ordonnances, et les coutumes, et enfin la jurisprudence. Les coutumes n'acquièrent que difficilement un statut équivalent à la loi et manquent, du point de vue de la doctrine, d'autorité. Les efforts d'un grand nombre de commentateurs reflètent la recherche d'un droit civil commun, dans l'imbroglio des divers espaces juridiques. 
L'Université, elle-même, n'enseigne que le droit canon depuis le Moyen Âge, et, malgré les interdictions, dans certaines universités, le droit romain : interdit à Paris en 1217, il est enseigné à Toulouse, Orléans et de plus en plus étudié par les romanistes à la fin du Moyen Âge, puis par les Humanistes. Le mouvement de retour au droit romain pur mené par $\mathrm{Cujas}^{29}$, contre Bartole (1313-1357) et les glossateurs, est contemporain de la rédaction des coutumes et les controverses continuent d'animer les juristes quant à la place du droit romain et à ce qui constitue le droit commun de la France. Cependant il faut attendre 1679 pour qu'un édit autorise les universités à enseigner non seulement le droit canon, mais le droit romain et institue une chaire d'enseignement du droit français contenu dans les ordonnances et les coutumes ${ }^{30}$. C'est dire que les commentateurs des coutumes, aidés par la jurisprudence, ont réussi à donner forme, à partir de la bigarrure de droits ${ }^{31}$ que soulignait Etienne Pasquier au XVI ${ }^{\mathrm{e}}$ siècle, à un Corps de droit français. Donner forme à ce Corps dans la lignée du Corpus iuris civilis romain passe aussi par la reconnaissance de la langue française comme langue du droit, ce qui est rendu possible par la rédaction des coutumes - vaste travail d'écriture - et la politique royale, de l'ordonnance de VillersCotterêts (1539) à la création d'une chaire de droit français, suivant celle de l'Académie française en 1635. Droit français et langue française connaissent alors une dynamique commune au service de l'État ${ }^{32}$.

Ces remarques rapides nous aident à comprendre l'héritage que reçoivent les rédacteurs et commentateurs de coutumes. On peut dire que la glose telle qu'elle se met en place au $\mathrm{XII}^{\mathrm{e}}$ siècle et la page glosée se perpétuent, avec des variantes, après l'imprimerie, dans les commentaires de la coutume, alors qu'elle tend à disparaître dans la plupart des ouvrages savants. C'est bien d'un rapport au texte de droit comme d'un texte sacré qu'il s'agit. L'interprète-exégète travaille sur le texte, et à partir de lui sur une sédimentation de textes, une compilation de références; il en propose soit une mise en ordre par réorganisation de la matière traitée, soit une nouvelle interprétation. Au fil des commentaires de coutumes, on peut constater que certains des interprètes ont acquis le statut d'autorité ; Charles du Moulin en est le meilleur exemple qui voit ses remarques et ses avis figurer dans toutes les éditions commentées. L'interprète s'inscrit dans une généalogie de commentateurs, une lignée à laquelle il apporte sa pierre et dont il sait pertinemment qu'il ne sera pas le dernier. Il s'appuie sur des ancêtres, non dans une perspective historique, mais dans un présent du discours. La mémoire du droit est au présent, a-historique.

Comment se présentent la page type des éditions de la coutume et l'ensemble du texte?

Il faut distinguer les éditions du cahier de la coutume des commentaires. Les premières ne présentent pas de rupture radicale avec le livre médiéval ${ }^{33}$, d'autant que les caractères sont gothiques. Le texte est en continu, la page dense, avec très peu d'alinéas, de marques de division, à part quelques signes typographiques, par exemple pour indiquer l'opposition de l'assemblée à un article de la coutume ou pour marquer les titres des sections. En 1547, l'écriture s'aère, les alinéas sont plus nombreux, un résumé de l'article en corps de caractères plus petit apparaît dans les marges, ainsi que des majuscules ornées en tête des titres. La lisibilité et l'accès au texte sont encore améliorés dans l'édition de 1571, où les marges qui entourent le procès-verbal portent les numéros des articles dont il est question, et les articles cités sont imprimés en italiques.

51 Pour les commentaires, la mise en page est beaucoup plus élaborée. Les éditions présentent des points communs importants qui rendent les différences secondaires. 
52 La page d'un livre de commentaires de coutumes se présente ainsi : l'article de la coutume est imprimé en pleine page, en gros caractères, puis vient sur une ou deux colonnes le résumé des questions numérotées auxquelles le commentaire répondra, en italiques, puis commence le commentaire en romain, avec des références au droit romain, ou à d'autres coutumes, à d'autres commentateurs anciens ou modernes, des citations d'arrêts, avec des résumés en marge, des citations d'auteurs. La page est à elle seule une mémoire, un type de discours et un mode de raisonnement; mémoire d'un processus historique mis à plat, comme si le droit n'était pas de l'histoire, pas du temps. Il brouille la chronologie. L'épître en latin de l'édition des coutumes réformées de 1571, en appelant à la rescousse tous les ancêtres, fonde l'autorité de la coutume et l'inscrit dans le discours généalogique du droit. Les références sont là pour étayer la vérité et la force du texte. Les interprètes ont cette mission - Balde, disciple de Bartole, les appelait la milice sans armes; celle-ci est en action pour les coutumes qui ont, en principe à partir de la rédaction, acquis un statut nouveau et l'autorité de la loi.

53 Avec les commentaires, la mise en page reprend l'héritage de la glose. Apparue au XII siècle, elle encadre le texte biblique, le Décret de Gratien, ou les textes du droit romain, puis occupe presque toute la page avec deux colonnes de commentaire, le texte glosé étant écrit en plus gros. Progressivement la page se charge jusqu'à atteindre la saturation, car les gloses sont un processus vivant, continu. Les marges se couvrent d'indications, noms des autorités auxquelles le glossateur se réfère, définitions de termes cités, renvois qui permettent de se repérer plus facilement dans l'ensemble du texte. Ce dernier se comble de gloses entre les lignes, ou à la fin, ou marginales, de commentaires explicatifs des titres, de distinctiones très développées, de quaestiones, et de controverses.

Quelques rares glossateurs se sont tournés vers le droit féodal commenté à la lumière du droit romain : dès le XIII ${ }^{\text {e }}$ siècle un docteur bolonais étudie les Consuetudines feudorum ${ }^{34}$. La méthode sera reprise au $\mathrm{XVI}^{\mathrm{e}}$ siècle pour interpréter les coutumes. Quant aux textes français, ils sont privés de gloses, car ils ne sont pas intégrés dans l'enseignement médiéval, sauf exceptionnellement des textes de droit. Le Coutumier bourguignon est glosé fin XIv ${ }^{e}$ siècle, en latin, langue pérenne et a-temporelle de l'Écrit ${ }^{35}$, ce qui inscrit le droit coutumier dans la tradition. Avec l'imprimerie, le double registre typographique (caractères romains et italiques) et la variation des corps de caractères permettent d'identifier clairement la nature de chaque information sur la page, le texte de la coutume, le commentaire, les questions, les références, etc. Roger Laufer ${ }^{36}$ distingue page glosée / page annotée qui symbolise la modernité. La première glorifie le texte et l'enserre dans la tradition. La seconde donne la primauté au texte, en rejetant les notes dans les marges et en bas de page. La page des éditions de coutumes tente de mêler les deux, la glose avec les acquis de la technique et les transformations de la rationalité. Estce pour pallier cette difficulté de lecture du texte, qui a été commenté longuement article par article que le Coutumier de Picardie, à la suite du commentaire de Jean-Marie Ricard, imprime la coutume en continu, sans commentaire ni note, restituant enfin au texte son intégrité et sa logique. Car il est clair que les commentaires, en sédiments successifs, et toutes les informations annexes, font éclater le texte de la coutume, noyé dans les interprétations.

55 L'édition commentée par Jean Du Fresne commence par une longue préface (six pages infolio) sur les fiefs, située avant le texte de la coutume et qui s'organise en dix-huit points, dont la question de l'origine des fiefs, romaine, lombarde, etc., de leur diversité. Puis suivent le titre premier Des Fiefs, l'article premier, en pleine page, les questions en 
italiques (sur deux colonnes), le commentaire (pleine page) qui intègre la réflexion, en s'aidant parfois de l'autorité du droit romain, de la conférence avec d'autres coutumes, de la jurisprudence, des ordonnances. Ce sont aussi sur des questions épineuses, de très longues dissertations. Jean-Marie Ricard par exemple commente très longuement dans le chapitre Testaments l'article LV sur les formalités en se référant à l'article 84 de l'ordonnance de Blois, à plusieurs arrêts, à d'autres coutumes, en explicitant la terminologie et proposant des interprétations. Le Traité des donations (édition de 1685 et suivantes), consacré à un chapitre des coutumes et à une question centrale du droit civil, reprend même la terminologie des glossateurs. Le livre est divisé en chapitres, sections à l'intérieur desquelles sont rangées les distinctions, et gloses. Les livres atteignent un nombre de pages sans commune mesure avec les éditions du seul texte de la coutume : 98 feuillets pour l'édition in-4 $4^{\circ}$ de 1571, 741 pages pour le commentaire d'Adrien de Heu infolio de 1653.

56 L'ampleur du travail des commentateurs participe à l'affirmation d'un droit commun français en intégrant systématiquement la réflexion sur le texte de la coutume à une réflexion sur la jurisprudence.

\section{Le livre entre mémoire et histoire}

S'arrachant peu à peu au commentaire du seul texte de la coutume, le livre s'enrichit de nouveaux éléments déjà évoqués, et d'autres comme les Questions notables de droit, ou les arrêts des cours souveraines et enfin des Observations sur des questions qui ont trait à l'histoire de la coutume.

Le cas le plus intéressant pour la coutume du bailliage d'Amiens est la manière dont est traitée, dans les éditions successives, la coutume locale de Gerberoy. En effet, la coutume locale de Gerberoy n'a pas été reçue à la rédaction de 1507. Les lettres de commission de Louis XII au bailli d'Amiens en 1506 contenaient une clause sur les pairs ${ }^{37}$ les engageant à comparaître pour corriger les coutumes, sans préjudice pour leurs droits, bien qu'ils ne relèvent que du parlement de Paris, et pas du bailli. Leur comparution ne sera pas interprétée comme preuve de leur soumission au bailliage. L'évêque de Beauvais, vidame de Gerberoy, conteste. Il fait assembler les états de son vidamé de Gerberoy qui accorde la coutume locale, mais cette dernière est refusée par les commissaires. La même contestation se répète à la réformation de 1567 et aboutit à un résultat identique. La question n'est pas réglée pour autant. La justice est interpellée sur ce point, dès avant la réformation. Charles du Moulin et quelques doctes juristes du parlement de Paris tranchent à l'occasion d'un procès en 1543 : l'évêque de Beauvais, qui prétend au droit de chambellage sur ses vassaux dans le vidamé de Gerberoy au nom d'une coutume locale, est débouté et la saisie déclarée tortionnaire ${ }^{38}$.

Par la suite, d'autres procès aboutissent à plusieurs arrêts contradictoires. La question des droits particuliers de l'évêque de Beauvais, au nom d'une coutume locale du vidamé, suscite, par ailleurs, de nombreux commentaires que toutes les éditions (sauf celle d'Adrien de Heu) reprennent, bien que la coutume locale du vidamé de Gerberoy n'ait pas été homologuée et que selon Jean-Marie Ricard elle ne soit plus observée. Pourtant des procès ont lieu qui concernent cette prétendue coutume et c'est ce qui semble expliquer la présence des Observations générales sur la coutume d'Amiens (chez du Fresne) où il est traité du problème de Gerberoy, et à cette occasion du rapport coutume générale-coutume locale. L'édition de 1661 du commentaire de Jean-Marie Ricard va plus loin, elle présente 
un sommaire particulier concernant cette coutume, alors que le titre général de l'ouvrage ne la mentionne pas, et les divers articles de la coutume locale de Gerberoy. On y trouve aussi le Discours concernant ce qui s'est passé au sujet des deux rédactions à propos de Gerberoy, qui sera repris dans toutes les éditions ultérieures et dans le Coutumier de Picardie où il est complété par des extraits des commentaires de Du Fresne et par les divers arrêts contradictoires portés sur la question. La raison invoquée pour l'édition est la suivante : la coutume a passé en non-usage avec le temps et ne l'a t-on plus considérée pour ce qui concerne le droit public, mais suivant un arrêt, elle

[...] sert encore pour les droits des seigneuries, entre Mgr l'évêque et les vassaux du vidamé et que d'ailleurs cette coutume contient d'assez belles dispositions pour les droits publics qui servent à l'éclaircissement des coutumes d'Amiens, de Senlis, et de Normandie, entre lesquelles elle se trouve située, j'ai cru que je rendrais service au public, en la faisant imprimer

avec les coutumes générales du bailliage. Bien que, ajoute-t-il, elle contienne aussi des droits exorbitants.

Il est évident que la rédaction des coutumes n'a pas résolu tous les problèmes, c'est ce qui peut expliquer dans les éditions la présence du cas du vidamé de Gerberoy, qui par toutes les questions qu'il soulève, suscite la réflexion des interprètes. Jean Du Fresne commentait en affirmant qu'une coutume locale non écrite (c'est le cas de celle de Gerberoy) n'est recevable en preuve de témoins et doit être par écrit, ce qui rejoint l'arrêt du 7-1-1608. Mais l'évêque obtient l'annulation de la décision en 1618, puis en 1621 un nouvel arrêt confirme la saisie féodale faute de relief payé, car elle est fondée en titre, sans qu'il soit besoin d'alléguer une coutume locale ou particulière. C'est dire que la cour reconnaît à l'évêque des droits supérieurs à ceux qui sont inscrits dans la coutume générale. L'acharnement des grands féodaux à défendre leurs privilèges et leurs droits particuliers rend compte de la tension permanente qui existe depuis la rédaction des coutumes sur la défense des droits seigneuriaux. Les questions touchant au droit féodal alimentent en grande partie la jurisprudence et, on le voit, les réponses sont contradictoires et difficiles à donner. Elles donnent lieu à toute une littérature juridique qui se consacre à l'un des chapitres des coutumes, le droit des fiefs.

Un léger glissement s'opère dans l'édition de 1781 d'Abbeville, renvoyant la question à l'histoire : l'intitulé change, on passe de Discours à Précis historique, sur ce qui s'est passé. Il s'agit toujours du commentaire (augmenté) de Jean-Marie Ricard, mais dans un livre renouvelé, édité par Dévérité, libraire imprimeur du roi et de Monseigneur Comte d'Artois. Louis Alexandre Dévérité (1743-1818) est actif comme libraire vers 1775. Fils du libraire Alexandre Dévérité, il est avocat au présidial d'Abbeville, membre de l'académie d'Amiens, auteur de brochures et d'ouvrages politiques et historiques, dont un Essai sur l'histoire de la Picardie, paru en 1770. Il sera quelques années plus tard officier de la garde nationale en 1790, député de la Somme à la Convention, puis entrera au Conseil des Anciens, et enfin juge au tribunal civil d'Abbeville sous l'Empire ${ }^{39}$.

La préface qu'il rédige pour présenter l'édition du commentaire de Jean-Marie Ricard est d'un ton très différent de tout ce qui a précédé et annonce son implication dans la Révolution. C'est un homme politique qui parle, un homme des Lumières qui considère la coutume d'une tout autre manière que les précédents commentateurs. Faut-il vraiment interpréter la coutume? La question en elle-même tranche radicalement avec ses prédécesseurs. Il se demande si de nouvelles remarques sur les coutumes se justifient, car tout commentaire ajouté à la loi est toujours une accusation portée contre elle, dans la mesure où cela induit que la loi est louche, douteuse, erronée, ambiguë, imprécise. D'autre 
part, plus les commentaires se multiplient, plus ils risquent d'étouffer le texte. Dans les autorités et les références citées, la nouveauté est grande qui mêle les Anciens et les Modernes, et le monde européen : d'Alembert, avec Solon, Aristote, les Anglais, le reste de l'Europe, Montesquieu, d'Argenson. Critique vis-à-vis de la coutume, Dévérité se demande quelles sont les raisons pour défendre ou non telle ou telle coutume, et justifie l'édition de celle d'Amiens par le fait qu'elle est une coutume d'égalité et de libre disposition et qu'elle favorise le commerce et les arts dans son ressort plus que ne le fait celle du Ponthieu ${ }^{40}$, par exemple.

Il s'éloigne de l'interprétation stricte du droit coutumier, telle qu'elle s'exprimait dans les éditions antérieures, et aborde l'histoire des mutations sociales, l'évolution de la noblesse, l'apparition de nouvelles formes de richesse dans leurs rapports avec la coutume et la législation. C'est un discours du changement et non plus de la tradition. La coutume est déjà presque entrée dans l'histoire.

De cette étude, plusieurs conclusions peuvent être tirées. La demande du public est toujours aussi vive pour les éditions de coutumes et surtout pour les commentaires à la veille de la Révolution, car ces derniers participent de la recherche d'un droit commun français, dont la nécessité s'impose, sans pouvoir réellement aboutir. Les rédactions ne sont pas venues à bout de toutes les difficultés. Le livre est autonome par rapport au cahier de la coutume, il autorise des découpages, des choix, et on l'a vu, en publiant par exemple des coutumes abrogées, il est aussi mémoire pour l'histoire. Si le livre prend quelque liberté avec le cahier et le texte de la coutume, c'est pour insérer la coutume dans la tradition, pour l'inscrire dans le champ de l'interprétation, pour l'articuler avec la jurisprudence, c'est-à-dire pour fonder son autorité en la référant à d'autres sources du droit. L'objectif recherché depuis les réformations est d'affirmer un corps du droit français, malgré la diversité coutumière qui demeure problématique. Le moyen d'y parvenir est dans un premier temps de gloser sur la coutume au même titre que sur le droit romain, puis d'élaborer une doctrine qui prenne en compte, voire qui transcende, cette hétérogénéité coutumière. En multipliant les conférences avec les autres coutumes, en traitant de chapitre particulier du droit coutumier d'une manière transversale sous forme de traité, les commentateurs renforcent ces grandes catégories du droit civil. La limite essentielle qu'ils rencontrent à la construction d'un corps de droit français réside dans le caractère local, irréductible, de la coutume.

\section{Annexe}

\section{Liste des éditions des coutumes du bailliage d'Amiens tirée de la Bibliographie des coutumes de France (André Gouron et OdileTerrin)}

Coustumes generalles du bailliage damiens, avec celles des prevostez de Montroeul, Beauquesne, Foulloy, Saint Ricquier, Doullens et Beauvoisis. Nouvellement publiées et décrétées en la ville damiens, par messieurs les commissaires déléguez de par le Roy nostre souverain seigneur sur le faict des Coustumes du royaulme de France. Imprimées par lordonnance des dits commissaires par Nicolas Le Caron demourant en ladite ville Damiens en la rue des Lombars, Amiens, s.d. In- $8^{\circ}$, car. goth., $95 \mathrm{ff}$.

Coustumes generalles du bailliage damiens avec celles des prévostez de Montroeul, Beauquesne, Foullois, Saint Ricquier et Beauvoisis. Nouvellement publiées et décrétées 
par messieurs les commissaires déléguez de par le Roy nostre souverain seigneur sur le faict des Coustumes du royaulme de France. A la fin: Imprimé à Paris pour Guillaume Eustace, libraire du Roy, demourant en la rue neufve Nostre Dame à l'Agnus Dei ou au Palays au troisième pillier lan mil cinq cens et seize, Paris, 1516. In-8 ${ }^{\circ}$, car. goth., $96 \mathrm{ff}$.

Coustumes generalles du bailliage damiens... On les vend à Paris à la rue neufve Nostre Dame à lagnus Dei et au pallays au troisième pillier. A la fin: Imprimé à Paris pour Guillaume Eustace, libraire du Roy, demourant... Paris, 1535. In-8 ${ }^{\circ}$, car. goth., $96 \mathrm{ff}$.

Coustumes generalles du bailliage Damyens avec celles des prevostez de Montroeul, Beauquesne, Foulloys, Saint Ricquier, Doullens et Beauvoisis nouvellement publiées et décrétées en la ville Damyens par messieurs les commissaires déléguez de par le Roy nostre souverain seigneur, sur le faict des Coustumes de France. On les vend à Amyens à la haute rue nostre dame près Sainct Martin chez Jehan Caron libraire. A la fin: Fin des Coustumes du bailliage Damyens nouvellement augmentées, additionnées et corrigées oultre les précédentes imprimées pour Jehan Caron... Amiens, 1547. In- $8^{\circ}$, car. goth., $114 \mathrm{ff}$.

Coustumes tant générales que locales et particulières du bailliage d'Amiens, mises et rédigées par escript de l'advis des trois estat dudict bailliage par nous Christophe de Thou, chevalier conseiller du Roy en son privé conseil et premier président en sa cour de Parlement, Barthélemy Faye et Jacques Viole, aussi conseillers dudict seigneur en sa dicte cour, commissaires à ce députez. Paris, pour Jean Dallier, 1571. In- $4^{\circ}, 16 \mathrm{ffnc}$. et $98 \mathrm{ff}$. 1575. Paris, pour la veufve Nicolas Roffet. In $-4^{\circ}, 16 \mathrm{ffnc}$. Et $98 \mathrm{ff}$.

1613. Amiens, imprimerie de Jacques Hubault. In-16, 24 ffnc. Et $143 \mathrm{ff}$.

\section{Coutumes commentées}

Heu (Adrien de), sieur de Conty, Les coustumes générales du bailliage d'Amiens commentées par Adrien d Heu, écuyer, sieur de Conty, conseiller du Roy en ses conseils d'Estat et Privé, président et lieutenant général en la séneschaussée de Ponthieu, et au présidial d'Abbeville, auparavant conseiller audit bailliage et siège présidial d'Amiens, avec une ample table des matières et questions traitées au présent commentaire, publiées après sa mort par Nicolas de Boulongnes, procureur du Roi à Abbeville. Paris, chez Gervais Alliot, 1653. In-fol., XIV-741 p. et table.

Du Fresne (Jean), Commentaire sur la coustume générale du bailliage d'Amiens et sur la coustume locale de la ville, prévosté et banlieue dudit Amiens, où sont rapportez les arrests donnez sur l'interprétation d'icelles depuis cinquante ans et plus. Avec une explication par forme de conférences de plusieurs articles, des coustumes de Ponthieu, Péronne et Arthois voisines, qui sont aussi pays de nantissement, par M. Jean Du Fresne, advocat en la cour de Parlement. Paris, imprimerie Estienne Maucroy, se vend chez Henry Le Gras, 1662. In-fol., X-478-84 p. et la table.

1662. Paris, chez Michel Bobin et Nicolas Le Gras. In-fol.

1671. Paris, idem.

Ricard (Jean-Marie), Coutumes tant générales que locales et particulières du bailliage d'Amiens, avec les notes de Maistre Charles Du Molin, et autres remarques particulières de M. Jean-Marie Ricard, avocat au Parlement. Paris, Louis Billaine, 1661. In-12, $\mathrm{X}-618-24 \mathrm{p}$. 
1683. Paris, chez la veuve de Robert Hubault. In-12, 454 p. et la table

Coutumes du bailliage d'Amiens, tant générales que locales et particulières avec des notes de Maître Charles Du Moulin, et autres remarques particulières par M. Jean-Marie Ricard, avocat au Parlement, augmentées de plusieurs autres nouvelles remarques. Paris, Guillaume Cavelier, 1712. In-fol., II-114 p. et la table.

Coutumes du bailliage d'Amiens commentées par Jean-Marie Ricard, nouvelle édition augmentée, Abbeville, Dévérité, 1781. In-12-, 83-292 p. et la table. (se trouve à la Réserve Pavillon de la bibliothèque de la Faculté de droit, Cujas)

Traité des donations entre vifs et testamentaires par Me Jean-Marie Ricard, avocat au Parlement, nouvelle et dernière édition augmentée par l'auteur avant sa mort, Paris Jean Guignard et Arnoul Seneuze, 1685.

Traité des donations entre vifs et testamentaires par Jean-Marie Ricard... avec la Coutume d'Amiens commentée par le même auteur. Dernière édition... Ensemble la Coutume de Senlis, commentée par le même auteur. Paris, Michel Guignard et Claude Robustel, 1713. 2 vol. in-fol.

1729-1730-1734. Paris, Nicolas Gosselin. 2 vol. in-fol.

Traité des donations... avec la Coutume d'Amiens commentée... Nouvelle édition, augmentée du traité de révocation des donations par la naissance ou survenance des enfans... (par Arnaud de La Rouvière)... Paris, Claude Robustel, 1734. 2 vol. in- $8^{\circ}$.

1734. Paris, M.-E. David. 2 vol. in-fol.

Traité des donations... avec la Coutume d'Amiens..., le traité de la révocation des donations..., ensemble la Coutume de Senlis commentée et les nouvelles additions aux œuvres ou traités de M. Jean-Marie Ricard, par M. Michel Du Chemin, avec une collection chronologique des ordonnances, édits, déclarations. Paris, Knapen, 1754. 2 vol. in-fol.

1783. Clermont-Ferrand et Riom, Degoutte, 2 vol. in-fol.

Recueil des commentateurs anciens et modernes sur les coutumes générales et particulières de France avec leurs textes corrigés, t. I ${ }^{\mathrm{er}}$. Coutumier de Picardie. Paris, aux dépens de la société, 1726. 2 vol. in-fol.

8 Calonne (Charles Alexandre), Observations et jugements sur les Coutumes d'Amiens, Artois, de Boulogne et de Ponthieu, sur plusieurs matières de droit civil et coutumier, par M. Calonne... Paris, P.-J. Simon, 1784. In-4 ${ }^{\circ}$, XII-568 p. (pas consulté, il n'est ni à la BNF ni à la bibliothèque de la faculté de droit).

\section{NOTES}

1. Roger Chartier et Henri-Jean Martin (dir.), Histoire de l'édition française, Paris, Fayard/Cercle de la Librairie, 1989, t. I ${ }^{\text {er }}$ : Le Livre conquérant. Du Moyen Âge au milieu du XVII siècle, 793 p., p. 551.

2. Nouveau coutumier général, ou corps des coutumes générales et particulières de France et des provinces connues sous le nom des Gaulles, exactement vérifiées sur les originaux conservés au greffe du parlement de Paris et des autres cours du royaume, avec les notes de MM. Toussaint Chauvelin, Julien Brodeau et Jean- 
Marie Ricard... jointes aux annotations de MM. Charles Du Molin, François Ragueau et Gabriel Michel de La Rochemaillet. Mis en ordre et accompagné de sommaires en marges des articles; d'interprétation des dictions obscures employées dans les textes, de listes alphabétiques des lieux régis par chaque coutume; et enrichi de nouvelles notes tirées des principales observations des commentateurs, et des jugements qui ont éclairci, interprété ou corrigé quelques points et articles des coutumes. Par Charles Bourdot de Richebourg, avocat au Parlement, Paris, Michel Brunet, 1724, 4 vol., in-fol.

3. Antoine Coron, «Ut prosint aliis. Jacques Auguste de Thou et sa bibliothèque ", in Claude Jolly (dir.), Histoire des bibliothèques françaises. Les bibliothèques d'Ancien Régime, 1530-1789, Paris, Promodis-Éditions du Cercle de la Librairie, 1988, t. I ${ }^{\mathrm{er}}, 547$ p., p. 101-125. Il s'agit du fils (1553-1617) de Christofle de Thou, qui fut chargé de la réformation des coutumes dans le ressort du Parlement de Paris. Le catalogue de sa bibliothèque fut édité par Joseph Quesnel, en 1679 à Paris. Yannick Nexon, « La bibliothèque du chancelier Séguier », ibid., p. 147-155.

4. Jean-Dominique Mellot, L'Édition rouennaise et ses marchés (vers 1600-vers 1730). Dynamisme provincial et centralisme parisien, Paris, École des Chartes, 1998, 816 p., p. 667-671.

5. La Face cachée du livre médiéval. L'histoire du livre, vue par Ezio Ornato, ses amis et ses collègues, Rome, Viella, 1997, 679 p. : contribution de Dominique Coq et Ezio Ornato, « La production et le marché des incunables. Le cas des livres juridiques ", p. 227-243.

6. Introduction de l'article d'Anette Smedley-Weill et de Simone Geoffroy-Poisson, supra, p. 13-92.

7. René Filhol, Le Premier Président Christofle de Thou et la réformation des coutumes, Paris, Librairie du Recueil Sirey, 1937, 301p., p. 47-48, et René Filhol, Le Vieux Coutusmier de Poictou, Bourges, É ditions Tardy, Travaux de la société d'histoire du droit et des institutions des pays de l'Ouest de la France, 1956, vol. I ${ }^{\mathrm{er}}, 329$ p. Les nobles émettent leurs doléances après la rédaction bâclée de 1514: «il y a eu de la malice par ceulx qui ont faict imprimer ce qui est imprimé, veu les obmissions qui ont esté faictes de tous les tiltres concernans les juridictions... »; voir également les problèmes concernant la réformation des coutumes dans l'article de Robert Descimon, infra, p. 13-107.

8. Pierre Aquilon, "Les réalités provinciales ", in Roger Chartier et Henri-Jean Martin, op. cit., p. 436-454.

9. C'est une région " en état d'alerte permanent » au XVI ${ }^{\mathrm{e}}$ siècle, Isabelle Paresys, Aux marges du royaume: violence, justice et société en Picardie sous François $1^{\text {er }}$, Paris, Publications de la Sorbonne, 1998, 396 p., p. 10.

10. Gustave Dupond-Ferrier, Les Officiers royaux des bailliages et sénéchaussées et les institutions monarchiques locales en France à la fin du Moyen Âge, Genève, Slatkine, 1974 (1 ${ }^{\text {re }}$ édition 1902), 1043 p., p. 37.

11. André Gouron, Odile Terrin, Bibliographie des coutumes de France. Éditions antérieures à la Révolution, Genève, Droz, 1975, 297 p. (voir les annexes de cet article).

12. Répertoire bibliographique des livres imprimés en France au seizième siècle, Baden-Baden, Librairie Valentin Koerner, 1971, $9^{\mathrm{e}}$ livraison, Amiens, p. 5-12.

13. Treize éditions de coutumiers jusqu'en 1552, dont certains contiennent aussi des coutumes non accordées, puis treize éditions avec des commentaires de Charles du Moulin, et d'autres commentateurs, jusqu'en 1681 et enfin Le Nouveau Coutumier de Charles Bourdot de Richebourg.

14. Alexandre Bouthors, Coutumes locales du bailliage d'Amiens, rédigées en 1507, Amiens, Mémoires de la société des antiquaires de Picardie, 1845-1853, t. I - II ; A.- I. Mamier, Ancien Coutumier inédit de Picardie, contenant les coutumes notoires, arrêts et ordonnances des cours, assises et autres juridictions de Picardie au commencement du quatorzième siècle (1300-1323), Paris, Techener, 1840.

15. Roger Chartier, "Stratégies éditoriales et lectures populaires, 1530-1660", in Histoire de l'édition française, op. cit., p. 699-721. Voir aussi tous les travaux d'Albert Labarre, Le Livre dans la vie amiénoise du seizième siècle. L'enseignement des inventaires après-décès, 1503-1576, Paris-Louvain, Nauwelaerts, 1971, 496 p. ; "Imprimeurs et libraires d'Amiens aux XVI et XVII ${ }^{\mathrm{e}}$ siècles. Les 
Hubault et les Caron », Bulletin trimestriel de la société des antiquaires de Picardie, 1989, p. 204-215; "Imprimeurs et libraires d'Amiens aux $\mathrm{XVII}^{\mathrm{e}}$ et $\mathrm{XVIII}{ }^{\mathrm{e}}$ siècles ", Bulletin trimestriel de la société des antiquaires de Picardie, 1991, p. 57-90.

16. John Gilissen, La Coutume, Brepols, Turhout-Belgium, 1982, 122 p., ch. IV, «Éditions et édition », p. 101-108.

17. Annie Charon-Parent, «Le monde de l'imprimerie humaniste : Paris », in Histoire de l'édition française, op. cit., p. 280-302.

18. Bernard Barbiche, «Le régime de l'édition ", in Histoire de l'édition française, op. cit., p. 467-471.

19. Jean-Dominique Mellot, L'Édition rouennaise, op. cit., p. 693.

20. Dans le Coutumier de Picardie, le privilège du roi est demandé par l'avocat qui a conçu l'ouvrage «craignant que quelques libraires, imprimeurs et autres s'avisent de contrefaire cet ouvrage ».

21. Voir note 2.

22. Régine Robin, "Fief et seigneurie dans le droit et l'idéologie juridique à la fin du XVIII siècle ", Annales historiques de la Révolution française, 1971, t. XLIII, n 206, p. 554-602.

23. Jean-Baptiste Domat, Les Loix civiles dans leur ordre naturel, Paris, 1689-1694, 3 vol.

24. Ibid., p. 6.

25. Roger Chartier, "Le prince, la bibliothèque et la dédicace ", in Marc Baratin et Christian Jacob, Le Pouvoir des bibliothèques. La mémoire des livres en Occident, Paris, Albin Michel, 1996, 338 p., p. 204-223.

26. Les exemplaires des anciennes éditions de cette coutume sont devenus très rares et manquent au public, selon l'édition de 1781.

27. Louis Boullenois, Dissertations sur des questions qui naissent de la contrariété des lois et des coutumes, Paris, 1732, 546 p. ; préface de M. Bouhier in Les Coutumes du duché de Bourgogne, avec les anciennes coutumes tant générales que locales de la même province, non encore imprimées et les Observations de M. Bouhier, Dijon, 1742.

28. Les CEuvres de Guy Coquille, Paris, 1665, 2 tomes. t. II, Les Coustumes du pays et comté de Nivernais enclaves et exemptions d'iceluy, avec les annotations de Me Guy Coquille, sieur de Romenay, p. 2 : « les coutumes des provinces de France qu'on appelle coutumiers sont leur vray droit civil et commun et peuvent être appelées Droit écrit ", il en conclut que le droit romain n'est pas le droit commun et qu'il ne doit être allégué que pour la raison.

29. Pierre Legendre, "La France et Bartole ", in Pierre Legendre, Écrits juridiques du Moyen Âge occidental, Londres, Variorum Reprints, 1988, chap. VII. Voir aussi les travaux de Eduard Maurits Meijers sur la place du droit romain au Moyen Âge, Études d'histoire du droit. 3 : Le droit romain au Moyen Âge. L'enseignement du droit romain dans trois universités du xiiie siècle, publié par les soins de Robert Feenstra et Herman Frederick Wilhelm David Fisher, Leyde, Universitaire pers Leiden, 1959, IX-324 p.

30. Philippe Sueur, Histoire du droit public français, $X V^{e}-X V I I I^{e}$ siècle, Paris, Presses universitaires de France, 1989, t. I ${ }^{\mathrm{er}}, 440$ p., t. 2, 601 p., p. 146-149.

31. Cité par Pierre Legendre, «La France et Bartole », p. 136, Etienne Pasquier, Lettres, livre IX, lettre 1 (à Brisson).

32. Xavier Martin, «Langue française et droit coutumier en France à l'époque moderne ", in G. van Dievœt, Ph. Godding et D. van den Auweele (éd.), Langage et droit à travers l'histoire. Réalités et fictions, Leuven-Paris, Peeters, 1989, 250 p., p. 135-145. Il cite le premier titulaire parisien de la chaire de droit français, François de Launay, commentant les Institutes d'Antoine Loisel; il faudrait un "Digeste coutumier ", " un corps de droit français, où l'on put voir ensemble et avec ordre tout ce qui est répandu confusément dans nos ordonnances et dans nos coutumes...», p. 144.

33. Anne Zali (dir.), L'Aventure des écritures. La page, Paris, Bibliothèque nationale de France, 1999, 215 p., p. 41. 
34. Eduard Maurits Meijers, « Les glossateurs et le droit féodal », op. cit., p. 261-277

35. Geneviève Hasenohr, « Discours vernaculaire et autorités latines », in Henri-Jean Martin et Jean Vezin (dir.), Mise en page et mise en texte du livre manuscrit, Paris, Éditions du Cercle de la librairie-Promodis, 1990, 471 p., p. 289-315.

36. Roger Laufer, «L'espace visuel du livre ancien », in Roger Chartier et Henri-Jean Martin, Histoire de l'édition française, op. cit., p. 579-601.

37. Charles Bourdot de Richebourg, Nouveau coutumier général, op. cit., t. III, 2, p. 668.

38. Jean du Fresne, Commentaire sur la coutume générale du bailliage d'Amiens, Paris, 1662, p. 471 sqq.

39. Jean-Dominique Mellot et Elisabeth Queval, Répertoire d'imprimeurs libraires, XVI $I^{e} X V I I I^{e}$ siècle. État en 1995 (4 000 notices), Paris, Bibliothèque nationale de France, 1997, 719 p.

40. Point de vue prémonitoire aussi sur la question de l'accessibilité à la justice, « les habitants des banlieues font chercher la justice à douze lieues de chez eux », sur les difficultés que cause une géographie coutumière stupide: certains villages sont partagés entre plusieurs coutumes. Pierre Louvet dans son livre paru en 1618 abordait la question pour le Beauvaisis, particulièrement mal loti de ce point de vue : la confusion coutumière est poussée à l'extrême, trois ou quatre coutumes se partagent un village, coutumes d'Amiens, de Montdidier, de Senlis, de Clermont. Coustumes de divers bailliages observées en Beauvaisis, a scavoir de Senlis, Amiens, Clermont et Montdidier, conférées l'une à l'autre et à celle de Paris, Beauvais, chez Godfroy Valet, 1618. 